\title{
Mitochondrial dysfunction, oxidative stress, and neurodegeneration elicited by a bacterial metabolite in a C. elegans Parkinson's model
}

\author{
A Ray ${ }^{1}$, BA Martinez ${ }^{1}$, LA Berkowitz ${ }^{1}$, GA Caldwell ${ }^{1,2}$ and KA Caldwell ${ }^{*, 1,2}$
}

Genetic and idiopathic forms of Parkinson's disease (PD) are characterized by loss of dopamine (DA) neurons and typically the formation of protein inclusions containing the alpha-synuclein ( $\alpha$-syn) protein. Environmental contributors to PD remain largely unresolved but toxins, such as paraquat or rotenone, represent well-studied enhancers of susceptibility. Previously, we reported that a bacterial metabolite produced by Streptomyces venezuelae caused age- and dose-dependent DA neurodegeneration in Caenorhabditis elegans and human SH-SY5Y neurons. We hypothesized that this metabolite from a common soil bacterium could enhance neurodegeneration in combination with PD susceptibility gene mutations or toxicants. Here, we report that exposure to the metabolite in C. elegans DA neurons expressing human $\alpha$-syn or LRRK2 G2019S exacerbates neurodegeneration. Using the PD toxin models 6-hydroxydopamine and rotenone, we demonstrate that exposure to more than one environmental risk factor has an additive effect in eliciting DA neurodegeneration. Evidence suggests that PD-related toxicants cause mitochondrial dysfunction, thus we examined the impact of the metabolite on mitochondrial activity and oxidative stress. An ex vivo assay of $C$. elegans extracts revealed that this metabolite causes excessive production of reactive oxygen species. Likewise, enhanced expression of a superoxide dismutase reporter was observed in vivo. The anti-oxidant probucol fully rescued metabolite-induced DA neurodegeneration, as well. Interestingly, the stress-responsive FOXO transcription factor DAF-16 was activated following exposure to the metabolite. Through further mechanistic analysis, we discerned the mitochondrial defects associated with metabolite exposure included adenosine triphosphate impairment and upregulation of the mitochondrial unfolded protein response. Metabolite-induced toxicity in DA neurons was rescued by complex I activators. RNA interference (RNAi) knockdown of mitochondrial complex I subunits resulted in rescue of metaboliteinduced toxicity in DA neurons. Taken together, our characterization of cellular responses to the $S$. venezuelae metabolite indicates that this putative environmental trigger of neurotoxicity may cause cell death, in part, through mitochondrial dysfunction and oxidative stress.

Cell Death and Disease (2014) 5, e984; doi:10.1038/cddis.2013.513; published online 9 January 2014

Subject Category: Neuroscience

Parkinson's disease (PD) is associated with dopaminergic neurodegeneration. Pathologically, this disease involves accumulation of the alpha-synuclein ( $\alpha$-syn) protein within proteineous inclusions called Lewy Bodies. ${ }^{1}$ Current neurodegeneration research is focused on identification of the causative factors and underlying mechanisms. Around $10 \%$ of PD cases are caused by genetic factors. Unknown factors, including environmental exposures (heavy metals and agricultural chemicals such as paraquat and rotenone), are associated with most cases of parkinsonism. ${ }^{2-6}$ There is also a higher incidence of PD in rural areas where the odds ratio cannot be completely accounted for by the level of toxic exposures often encountered by the use of chemicals in farming. ${ }^{7}$ Associated with rural living, individuals exhibit a much greater interaction with the surrounding terrestrial environment through mechanisms such as well water consumption, farming, gardening, and/or living on dirt floors.

Streptomyces are a ubiquitous soil bacterial genus that have large genomes and produce a variety of secondary metabolites, including compounds that cause mitochondrial defects. ${ }^{8}$ Evidence suggests that PD-related toxicants cause oxidative stress and mitochondrial dysfunction, which can lead to parkinsonism in animals. ${ }^{9-11}$ In previous work, we reported that a bacterial metabolite produced by Streptomyces venezuelae caused age- and dose-dependent dopamine (DA) neurodegeneration in Caenorhabditis elegans and dose-dependent degeneration of human DA producing SH-SY5Y cells. ${ }^{12}$ Thus, this metabolite might represent a

\footnotetext{
${ }^{1}$ Department of Biological Sciences, The University of Alabama, Tuscaloosa, AL, USA and ${ }^{2}$ Departments of Neurobiology and Neurology, Center for Neurodegeneration and Experimental Therapeutics, University of Alabama at Birmingham, Birmingham, AL, USA

${ }^{*}$ Corresponding author: K Caldwell, Department of Biological Sciences, The University of Alabama, Tuscaloosa, AL 35487-0344, USA. Tel: +1 205 348 4021; Fax: + 1205348 1786; E-mail: kcaldwel@bama.ua.edu

Keywords: ROS; dopamine; neurotoxin; Streptomyces

Abbreviations: 6-OHDA, 6-hydroxydopamine; $\alpha$-syn, alpha synuclein; DA, dopamine; D $\beta H B$, D-beta hydroxybutyrate; EtAc, ethyl acetate; EV, empty vector; GFP, green fluorescent protein; PD, Parkinson's disease; RNAi, RNA interference; ROS, reactive oxygen species; UPR ${ }^{\mathrm{ER}}$, endoplasmic reticulum unfolded protein response; UPR ${ }^{\mathrm{mt}}$, mitochondrial unfolded protein response; UPS, ubiquitin proteasome system

Received 29.7.13; revised 18.11.13; accepted 20.11.13; Edited by E Baehrecke
} 
previously uncharacterized environmental contributor to neurodegeneration.

Here, we extend the mechanistic analysis of this novel environmental effector of neurodegeneration to report that exposure to the $S$. venezuelae metabolite causes excessive production of reactive oxygen species (ROS) in $C$. elegans, as shown by a cellular reporter for superoxide dismutase and an in vitro biochemical assay. Likewise, the mitochondrial unfolded protein response (UPR ${ }^{\mathrm{mt}}$ ) pathway was upregulated and adenosine triphosphate (ATP) production impaired in response to metabolite exposure. In combinational studies using additional chemical and genetic modifiers associated with PD, we determined that metabolite exposure enhanced susceptibility to cell death. Moreover, we discerned that the mechanism of action involves targeting of mitochondrial complex I, and that antioxidant treatment rescues DA neurodegeneration. Taken together, these data provide a plausible underlying mechanism involved in $S$. venezuelae metaboliteinduced toxicity.

\section{Results}

S. venezuelae metabolite exposure causes oxidative stress in $\boldsymbol{C}$. elegans. As previously reported, ${ }^{12}$ the neurotoxic compound under investigation is a small secondary product (molecular weight $<300$ ) isolated following growth of $S$. venezuelae to the stationary phase in liquid culture where the compound is present in spent media. The $S$. venezuelae conditioned medium was extracted in dichloromethane (DCM), and ethyl acetate (EtAc) solvent was used to reconstitute the compound following partitioning, indicating that it is amphipathic. We have calculated an almost $100 \%$ recovery rate from this extraction (data not shown). Hereafter, we use the term metabolite to refer to this compound. EtAc is used as a negative (solvent) control in all experiments and does not cause a significant DA neurodegeneration.

To determine whether the metabolite increases ROS production in vivo, we examined $C$. elegans expressing an established oxidative stress-inducible reporter, sod-3::GFP, where green fluorescent protein (GFP) is driven under the endogenous sod-3 gene promoter. ${ }^{13}$ sod-3 encodes a mitochondrial superoxide dismutase enzyme, which is thought to protect against oxidative stress. Worms treated with the metabolite exhibited a significant upregulation of sod-3::GFP expression compared with EtAc solvent control (Figures 1a-d). RNA interference (RNAi) depletion of daf-2, the $C$. elegans insulin/IGF receptor ortholog, was used as a positive control ${ }^{14}$ (Figures 1a and d).

ROS production in response to the metabolite was also examined in $C$. elegans using an ex vivo 2,7-dichlorofluorescein diacetate (DCF-DA) assay. ${ }^{15}$ Worms treated with either the $S$. venezuelae metabolite, $100 \mu \mathrm{M}$ paraquat (positive control), or EtAc solvent were analyzed at day 7 , day 10 , and day 12 of exposure because DA neurodegeneration is observed following metabolite exposure at these time points. $^{12}$ The results showed significantly increased ROS production in metabolite- and paraquat-exposed worms at all days analyzed. Figure 1e displays day 7 data (days 10 and 12 are not shown).
Previously, we reported that exposure to the $S$. venezuelae metabolite caused DA neurodegeneration. ${ }^{12}$ Treatment with $1 \mathrm{mM}$ probucol, an anti-oxidant, fully rescued metaboliteinduced DA neurodegeneration (Figures $1 \mathrm{f}-\mathrm{j}$ ). The protection by probucol indicates that free radical generation contributes to $S$. venezuelae metabolite toxicity. Thus, these data suggest that the $S$. venezuelae metabolite induces oxidative stress that, in turn, contributes to neuronal cell death.

Because sod-3 is under the direct control of DAF-16, we sought to determine whether the DAF-16 transcription factor could be induced to translocate to the nucleus in response to metabolite treatment. ${ }^{16}$ When compared with solvent treatment alone, we found that DAF-16 significantly accumulates within nuclei when animals are treated with metabolite or challenged with daf-2 knockdown (Figures $2 a$ and b). The nuclear accumulation observed could be related to increased ROS, because DAF-16 is a known stress-associated transcription factor induced by ROS, however, DAF-16 also responds to other stressors. ${ }^{17}$

In mammals, NRF-2 is the major ROS and detoxification transcription factor. ${ }^{18}$ The $C$. elegans NRF-2 homolog, $\mathrm{SKN}-1$, can be translocated to the nucleus by a variety of sources including pathogens and ROS. ${ }^{19,20}$ However, when put to direct observation, SKN-1 failed to change its intracellular localization in response to metabolite treatment (data not shown), indicating that the ROS produced by the metabolite in $C$. elegans may not be sensed by the SKN-1 machinery.

\section{S. venezuelae metabolite toxicity causes mitochondrial} dysfunction. Mitochondria are known to be a major source of ROS and perturbations that affect their metabolic activity can further promote the build-up of ROS and lead to complexities within the mitochondria such as an accumulation of misfolded proteins. ${ }^{21}$ This subsequently triggers a stress-response pathway referred to as the $\mathrm{UPR}^{\mathrm{mt}}$, which activates transcription of mitochondrial chaperone genes to promote protein homeostasis. In $C$. elegans, this can be monitored in a strain expressing $h s p-6:$ GFP, ${ }^{22}$ where HSP-6 is a nuclear encoded mitochondrial chaperone and GFP is driven by the $h s p-6$ promoter. $S$. venezuelae metabolite exposure caused an upregulation of $h s p-6:$ :GFP in day 4 worms when compared with solvent control (Figures $3 a-c)$. RNAi depletion of mev-1, a $C$. elegans ortholog of a mitochondrial electron transport chain complex II subunit, was used as a positive control for increased $h s p-6$ activation $^{23}$ (Figure 3a).

To investigate whether the $S$. venezuelae metabolite impacts mitochondrial respiratory chain activity (complex I-IV), ATP levels were measured in C. elegans extracts treated with either EtAc solvent control or $S$. venezuelae metabolite. A metabolic product of the neurotoxin MPTP [1-methyl-4-phenylpyridinium (MPP + )], which was shown to significantly decrease ATP production in a previous C. elegans study, was used as a positive control. ${ }^{24}$ Our results showed that worms exposed to the $S$. venezuelae metabolite or MPP + displayed significantly lower overall levels of ATP as compared with the solvent control (Figure 3d). Taken together, exposure to the $S$. venezuelae metabolite caused reduction in ATP levels, upregulation of 

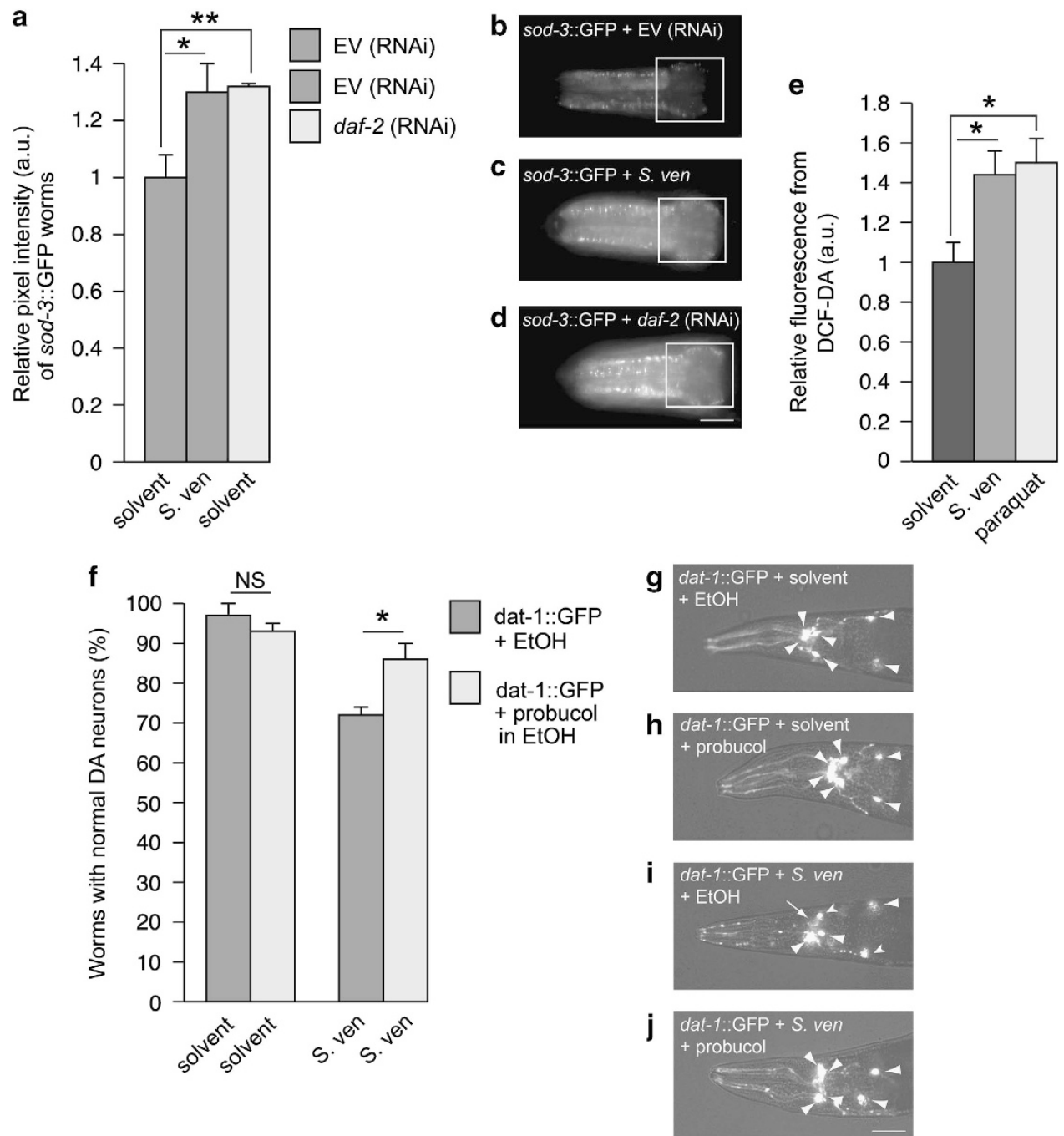

Figure 1 S. venezuelae metabolite causes oxidative stress in C. elegans. (a) S. venezuelae (S. ven) metabolite caused an upregulation of sod-3::GFP expression, an indicator of oxidative stress, in empty vector (EV) RNAi-treated worms when compared with worms exposed to solvent only, as quantitated using pixel intensities as described in (b-d). When compared with metabolite exposure, RNAi knockdown of daf-2, used as a positive control, expressed similar levels of sod-3::GFP. Values are the mean \pm S.D. of 3 experiments where 30 animals were analyzed per replicate $\left({ }^{*} P<0.05 ;{ }^{* \star} P<0.01\right.$; one-way ANOVA). The values were normalized to the untreated solvent control. (b-d) Representative worm images for each of the treatments described in (a) where pixel intensities were measured in a $100 \times 100 \mu \mathrm{m}$ region at the anterior bulb of the pharynx. The white box shows the region of GFP measured in all animals. (e) S. venezuelae metabolite and $100 \mu \mathrm{M}$ paraquat (positive control) significantly increased the amount of intracellular ROS compared with solvent control. Worms were evaluated using a DCF-DA assay by examining extracts $\left(^{*} P<0.01\right.$; one-way ANOVA; $n=3$ independent experiments). The values were normalized to the untreated solvent control. (f) Treatment with $1 \mathrm{mM}$ probucol (dissolved in ethanol), an anti-oxidant, significantly rescued the $S$. venezuelae-induced neurotoxicity in DA neurons compared with metabolite treatment alone $\left({ }^{*} P<0.01\right.$; Student's $t$-test; $n=3$ independent experiments). (g-j) Representative images of the probucol experiment described in (g). All C. elegans (strain BY200) express GFP specifically in the six anterior DA neurons. In all images, large arrowheads show intact dopaminergic neuron cell bodies. Arrows indicate areas where dopaminergic neurons have degenerated. Small arrowheads indicate cell body degeneration. (g) Exposure to EtAc and ethanol (solvent for probucol) did not result in DA neuron loss. (h) The addition of probucol did not cause neurotoxicity, as evidenced by intact DA neurons. (i) S. venezuelae metabolite exposure caused substantial degeneration of cell processes, as displayed throughout the processes. Further, two of the cell bodies are degenerating and one is missing in this representative worm. (j) Probucol rescues $S$. venezuelae-induced DA neuronal toxicity, as shown in this $C$. elegans example. Magnification bars $=50 \mu \mathrm{m}$

$\mathrm{UPR}^{\mathrm{mt}}$, and production of ROS, revealing a role in mitochondrial dysfunction. These properties of the $S$. venezuelae metabolite are mechanistically similar to other environmental neurotoxins such as rotenone and paraquat. ${ }^{9,11}$

\section{S. venezuelae metabolite toxicity involves mitochondria} complex I. Previously, it was demonstrated that the environmental toxins, rotenone and paraquat, as well as the experimental toxin model, 6-hydroxydopamine (6-OHDA), inhibit complex I of the mitochondria. ${ }^{11,25,26}$ Our results indicating that exposure to the $S$. venezuelae metabolite caused impairment of mitochondrial function led us to further investigate whether this metabolite targets complex I. Here, we explored whether two chemicals that rescue complex I deficiency would provide protection against $S$. venezuelaeinduced DA neurodegeneration. Riboflavin is an activator of mitochondrial complex I (NADH dehydrogenase) and D-beta-hydroxybutyrate $(\mathrm{D} \beta \mathrm{HB})$ is a complex II activator 


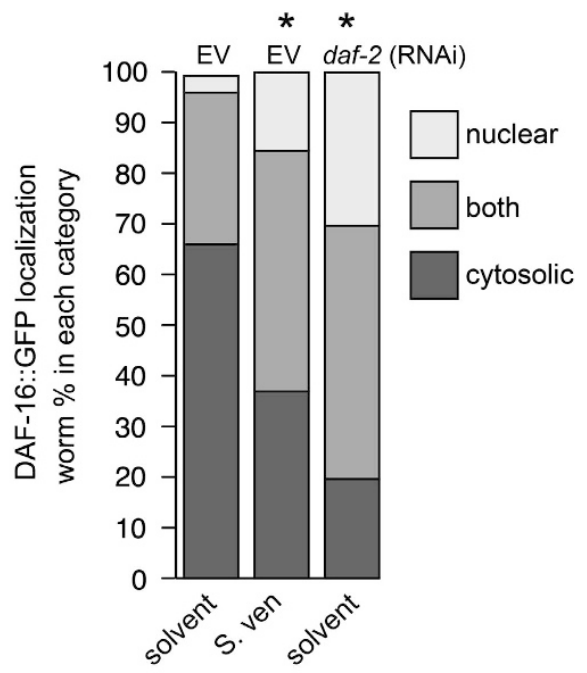

b

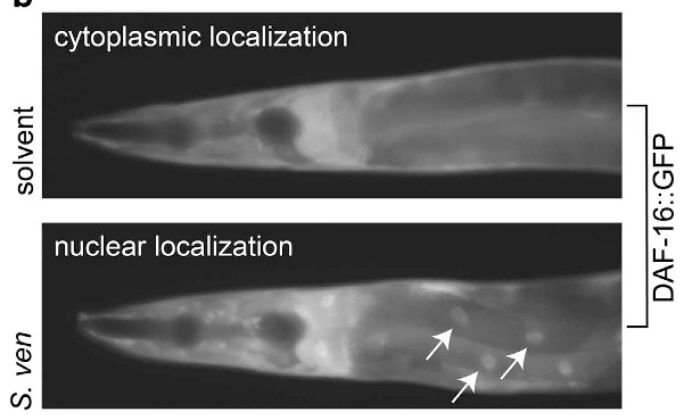

Figure 2 Effect of $S$. venezuelae metabolite on DAF-16 localization. (a) Stacked graph representing the percentage of $C$. elegans with DAF-16::GFP localization in the nucleus, cytoplasm, or both. $S$. venezuelae exposure promotes nuclear translocation of DAF-16::GFP in a manner similar to daf-2 (RNAi), where both treatments were significantly different from EV solvent control ${ }^{*} P<0.05$; one-way ANOVA; $n=3$ independent experiments with 30 animals/experiment). (b) Representative images of DAF-16::GFP localization in the cytoplasm and nucleus (arrows) following treatment with EtAc solvent and metabolite, respectively

(succinate dehydrogenase) that rescues complex I defects by a complex II-dependent mechanism. ${ }^{27-29}$ In these experiments, riboflavin treatment significantly rescued S. venezuelae neurotoxicity (Figures $4 a, c$, and d). Similarly, treatment with $\mathrm{D} \beta \mathrm{HB}$ significantly protected $\mathrm{DA}$ neurons from $S$. venezuelae metabolite-induced degeneration (Figures $4 \mathrm{~b}$, $c$, and e).

To further investigate the involvement of mitochondrial electron transport chain, we performed RNAi knockdown of mitochondrial genes, gas- 1 and nuo-1 (subunits of complex I) and mev-1 (subunit of complex II) specifically in DA neurons, and assayed for neurodegeneration using DA neuron-specific RNAi $^{30}$ Depletion of these gene products without metabolite did not result in DA neurodegeneration (Figure 4f). Notably, addition of metabolite also did not cause DA neurodegeneration in either of the complex I gene knockdown conditions while the metabolite did cause degeneration in mock, empty vector (EV) RNAi conditions. Therefore, this experiment provides evidence that complex $\mathrm{I}$ is impaired by the metabolite. Conversely, knockdown of the complex II subunit, $m e v-1$, resulted in a significant neurodegeneration compared with control (Figure 4f). Moreover, RNAi knockdown of complex I genes resulted in rescue of metabolite-induced DA neurodegeneration compared with mock RNAi treated with metabolite (Figure 4f). These results, together with the neuroprotection afforded by riboflavin and $\mathrm{D} \beta \mathrm{HB}$, suggest that complex I is involved in $S$. venezuelae metabolite-induced neurotoxicity. This complex I-specific effect could be due to the excessive production of ROS following metabolite exposure.

Mitochondrial complex I function may be decreased by $\alpha$-syn, ${ }^{31}$ a protein that when overexpressed or mutated, can lead to familial PD. Overexpression of $\alpha$-syn is often used to induce neurodegeneration in animal models. In C. elegans, dopaminergic neurons undergo age-dependent neurodegeneration following human $\alpha$-syn overexpression. ${ }^{32} \mathrm{We}$ exposed transgenic $\alpha$-syn worms to the metabolite and discovered that the DA neurons showed enhanced degeneration to metabolite treatment (Figures $5 \mathrm{a}-\mathrm{c}$ ). Furthermore, $\alpha$-syn-expressing worms exposed to metabolite displayed significantly more DA neurodegeneration than metabolitetreated animals expressing GFP only in DA neurons (Figure 5a). Thus, the compromised genetic background in the DA neurons of these animals rendered them more susceptible to secondary neurotoxicity via metabolite exposure.

In post-mortem human brains, mitochondrial accumulated $\alpha$-syn was shown to interfere with complex I. ${ }^{31}$ Furthermore, in vitro studies demonstrated that $\alpha$-syn directly associates with mitochondrial membranes and causes mitochondrial fragmentation. ${ }^{33}$ In this regard, we sought to evaluate whether there was an additive effect from the metabolite in neurons compromised from both $\alpha$-syn overexpression and reduced complex I or II function through RNAi knockdown. Without metabolite susceptibility to DA neurodegeneration was significantly enhanced following RNAi knockdown of any one of these three genes beginning at day 6 compared with EV control in $\alpha$-syn-expressing worms (Figure $5 \mathrm{~d}$ ). Metabolite exposure of $\alpha$-syn-expressing $C$. elegans that were also treated with gas-1 or mev-1 (RNAi) did not result in enhanced DA neurodegeneration (Figure 5d). However, metabolite exposure of these worms treated with nuo-1 (RNAi) resulted in enhanced neurodegeneration compared with nuo-1 (RNAi) solvent control worms. Also, DA neurodegeneration was exacerbated by nuo-1 (RNAi) versus EV control when treated with metabolite (Figure $5 \mathrm{~d}$ ). These data suggest that $\alpha$-syn-expressing DA neurons treated with metabolite treated are more vulnerable to nuo-1 knockdown. In general, mechanisms associated with $\alpha$-syn and complex I gene interactions are not fully understood. Thus, the effect observed with our results in Figure 5d (versus Figure 4f) could be a result of direct or indirect interactions between metabolite, $\alpha$-syn and mitochondrial components.

Another familial form of PD, mutation in LRRK2 (G2019S), has been shown to impact mitochondrial function whereby mitochondrial membrane potential and intracellular ATP production were impaired. ${ }^{34}$ Studies in nematodes and mice have shown that this LRRK2 mutation can increase kinase activity and cause neuronal toxicity. ${ }^{35}$ Thus, we examined the effect of metabolite using the LRRK2 G2019S mutation in C. elegans. The metabolite significantly enhanced DA neurotoxicity compared with solvent control (Figure 5e). 
a

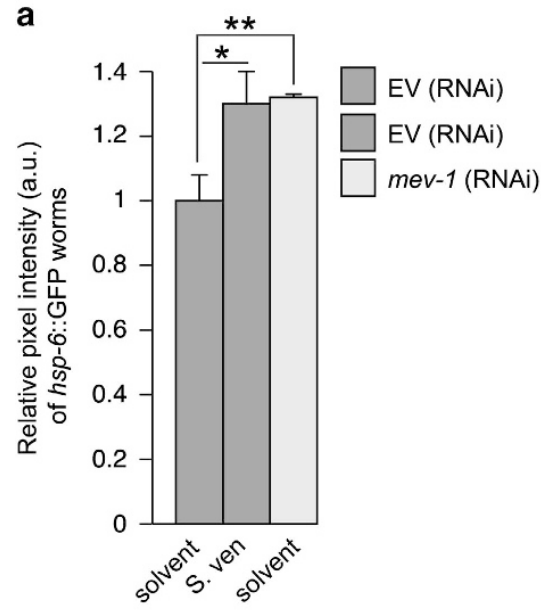

C

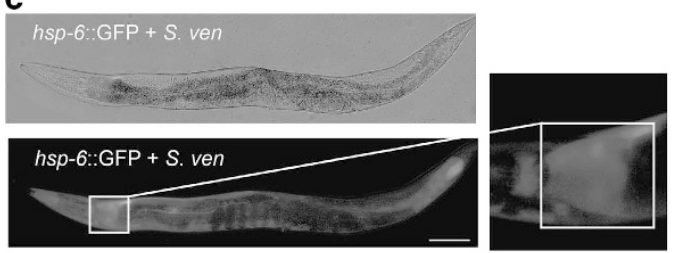

b
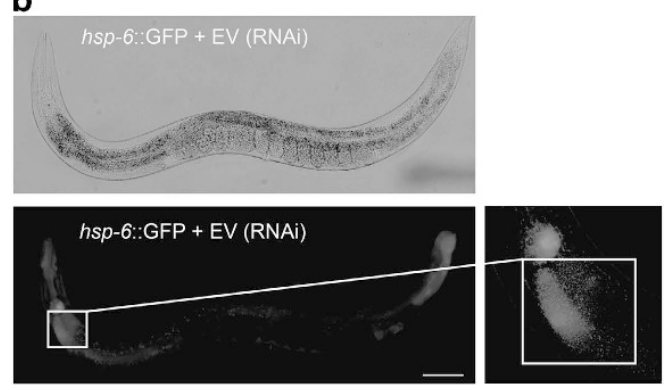

d

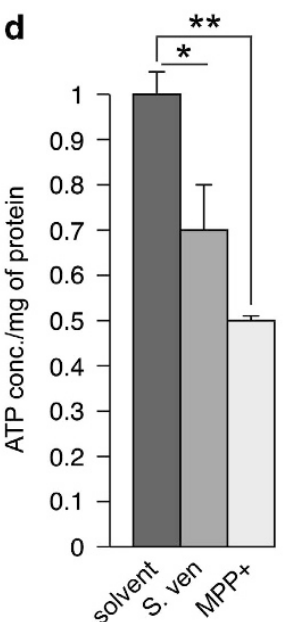

Figure 3 S. venezuelae metabolite causes mitochondrial dysfunction. (a) S. venezuelae metabolite caused an upregulation of $h s p-6:: G F P$, an indicator of UPR ${ }^{\mathrm{mt}}$ stress response. In this experiment, expression of $h s p-6:$ GFP in EV RNAi-treated worms exposed to metabolite was significantly increased compared with worms exposed to solvent only, as quantitated using pixel intensities as visualized in (b and $\mathbf{c}$ ). When compared with metabolite exposure, RNAi knockdown of mev-1, used as a positive control, expressed similar levels of $h s p-6::$ GFP. Values are the mean \pm S.D. of 3 experiments where 30 animals were analyzed per replicate $\left({ }^{*} P<0.05 ;{ }^{* \star} P<0.01 ;\right.$ one-way ANOVA). The values were normalized to the untreated solvent control. ( $\mathbf{b}$ and $\mathbf{c}$ ) Representative worm images (brightfield (top) and fluorescence (bottom)) for EtAc and metabolite treatments described in (a). Pixel intensities were measured in a $100 \times 100 \mu \mathrm{m}$ region in the intestinal lumen immediately posterior to the grinder of the pharynx. The region highlighted in the white box shows GFP expression driven by the $h s p-6$ gene promoter that was measured in all animals and is magnified to the right. (d) $C$. elegans exposed to the $S$. venezuelae metabolite showed reduced ATP production compared with the solvent control; worms treated with $1 \mathrm{mM}$ MPP + , which is known to reduce ATP levels, also demonstrated a similar effect $\left({ }^{*} P<0.05 ;{ }^{* *} P<0.01\right.$; one-way ANOVA). Values are the mean \pm S.D. of three independent experiments and were normalized to the untreated control. Magnification bar $=100 \mu \mathrm{m}$

When exposed to metabolite, LRRK-2 G2019S expressing worms displayed significantly more DA neurodegeneration than animals expressing GFP only in DA neurons (Figure 5e).

\section{S. venezuelae metabolite potentiates 6-OHDA or rotenone-} induced DA neurodegeneration. Epidemiological studies indicate that idiopathic parkinsonism could result from a combination of various risk factors. Therefore, we asked whether exposure to toxicants used in PD models that induce mitochondrial dysfunction and oxidative stress, such as $6-O H D A$ and rotenone, ${ }^{36}$ along with the $S$. venezuelae metabolite, would result in enhanced DA neurodegeneration. 6-OHDA inhibits mitochondrial complexes I and IV, whereas rotenone displays specificity for complex I. 9,25

We first examined a combination of 6-OHDA and metabolite. $C$. elegans were incubated with the $S$. venezuelae metabolite for half the standard exposure time to enhance visualizing neuronal vulnerability to a secondary stressor, such as 6-OHDA, while not appreciably causing neurodegeneration with metabolite alone. In this metabolite-only exposure paradigm, $\sim 80 \%$ of the population still displayed normal DA neurons (Figures $6 \mathrm{a}$ and b). Nematodes were exposed to the metabolite (or EtAc solvent control) and exposed to $6-O H D A$ for $1 \mathrm{~h}$ at late larval stage 4 . Here, we observed that $48 \mathrm{~h}$ post 6-OHDA treatment, worms exposed to $S$. venezuelae metabolite and 6-OHDA did not show a significant degeneration compared with worms treated with 6 -OHDA alone. However, after $72 \mathrm{~h}$, this same treatment provided a significant degeneration with respect to the controls (Figures 6b-e).

We further investigated the combined effect of rotenone, a known complex I inhibitor, and $S$. venezuelae metabolite. Unlike the 6-OHDA regimen, worms were continuously exposed to rotenone or DMSO solvent from the L4 stage and analyzed at day 10 (Figure 6f). Co-exposure of the metabolite with rotenone enhanced DA neurodegeneration compared with metabolite or rotenone alone (Figures $6 \mathrm{~g}-\mathrm{i}$ ). Together, these results suggest that mitochondrial respiratory chain activity is sensitive to the metabolite-induced toxicity. These data also suggest that exposure to more than one environmental/chemical risk factor has an additive effect on DA neurodegeneration. 
a

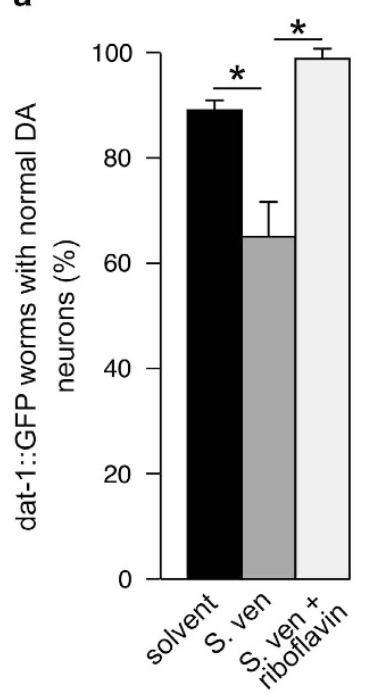

b

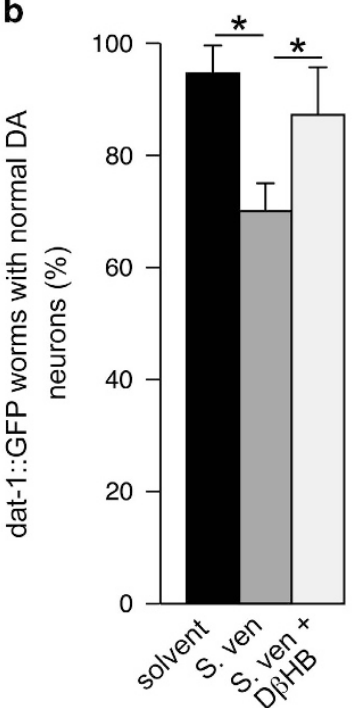

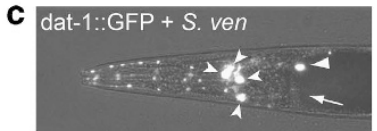

d dat-1::GFP + S. ve

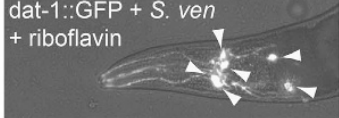

e dat-1::GFP + S. ven $+\mathrm{DBHB}$

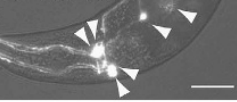

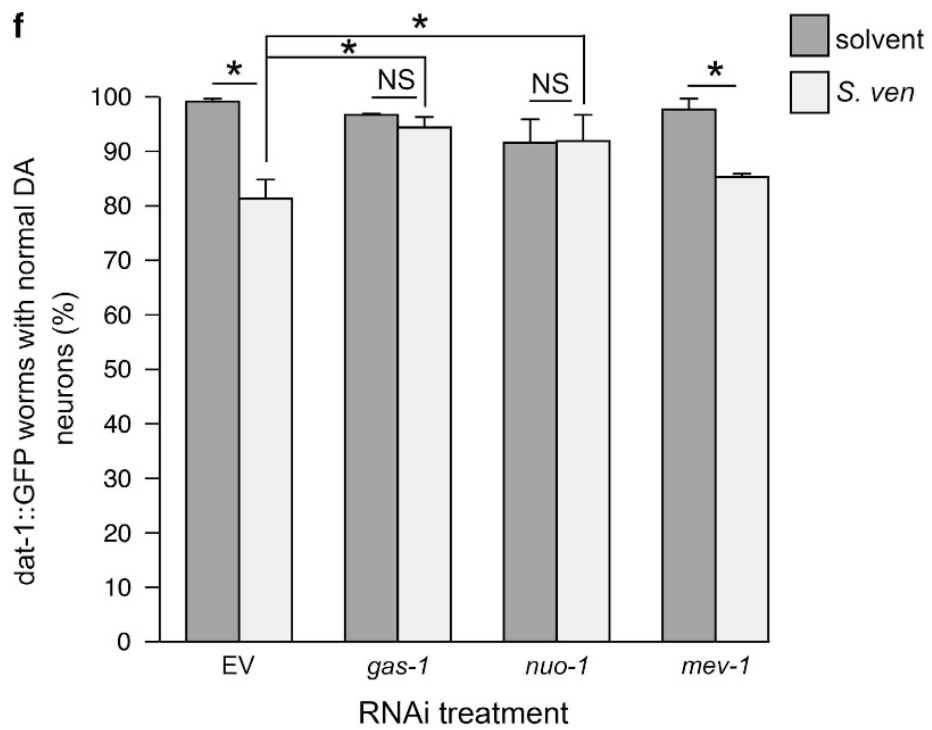

Figure 4 The S. venezuelae metabolite impacts mitochondrial complex I. (a-e) Metabolite-induced DA neurotoxicity was rescued by riboflavin and D $\beta H B$, drugs that rescue mitochondria complex I deficiency. (a) $S$. venezuelae and $1 \mu \mathrm{g} / \mathrm{ml}$ riboflavin significantly rescued DA neurons compared with the metabolite alone. (b) When $50 \mathrm{mM}$ $\mathrm{D} \beta \mathrm{HB}$ is co-administered with the metabolite, $C$. elegans $\mathrm{DA}$ neurons are rescued from neurotoxicity. (c-e) Representative images of riboflavin and $\mathrm{D} \beta \mathrm{HB}$ rescuing $\mathrm{DA}$ neurotoxicity induced by the metabolite. All C. elegans (strain BY200) express GFP specifically in the six anterior DA neurons. In the images, large arrowheads show intact dopaminergic neuron cell bodies. Arrows indicate areas where dopaminergic neurons have degenerated. Small arrowheads indicate cell body degeneration. (c) Exposure to the $S$. venezuelae metabolite caused neuronal loss in this worm where five of the six DA neurons are degenerating. (d) The addition of riboflavin completely rescued DA neurons in an animal exposed to the metabolite. (e) $\mathrm{D} \beta \mathrm{HB}$ also rescued $S$. venezuelae-induced DA neuronal toxicity, as shown in this $C$. elegans example. Magnification bar $=50 \mu \mathrm{m}$. (f) RNAi knockdown of complex I components gas-1 and nuo-1 resulted in rescue of DA neurodegeneration when treated with metabolite while complex II component mev-1 and EV RNAi showed enhanced DA neurodegeneration with metabolite exposure ( ${ }^{*} P<0.05$; one-way ANOVA; $n=90$ worms). C. elegans (strain UA202) were analyzed at day 12 where data were analyzed as the mean \pm S.D.

\section{Discussion}

We previously reported an initial description of a highly stable and small molecular metabolite from $S$. venezuelae that caused $C$. elegans dopaminergic neurodegeneration and death of human SH-SY5Y neurons. ${ }^{12}$ The impetus for that nascent work stemmed from our interest in what type of hypothetical and more common exposure could, in combination with misfortune in 'the genetic lottery', potentially account for the prevalence of PD - the second most common neurodegenerative disorder. However, while multi-hit hypotheses are attractive in clinically defining disease etiology, there remains little functional evidence for such scenarios. In this more mechanistic investigation, we exploit the advantages afforded through $C$. elegans research to combine genetic and toxicological analysis in a well-defined system that facilitates rapid and reproducible evaluation of neurodegeneration. We determined that the neurotoxic $S$. venezuelae metabolite caused excessive production of free oxygen radicals, upregulated the $\mathrm{UPR}^{\mathrm{mt}}$, and impaired mitochondrial complex I activity (Figure 7). 
a
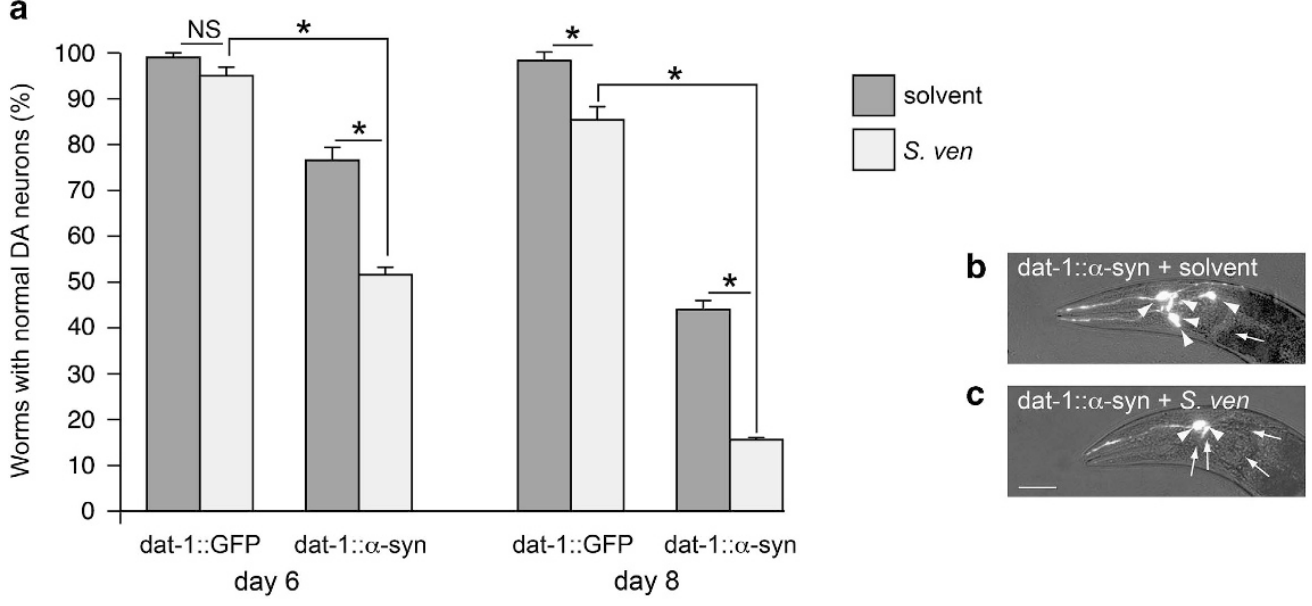

c

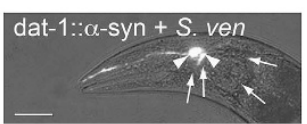

d

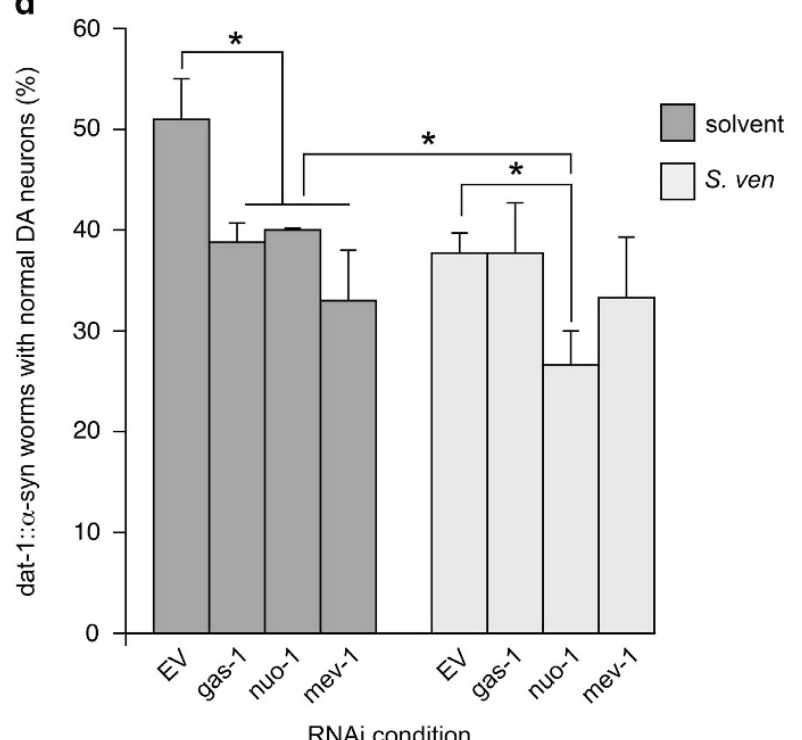

e

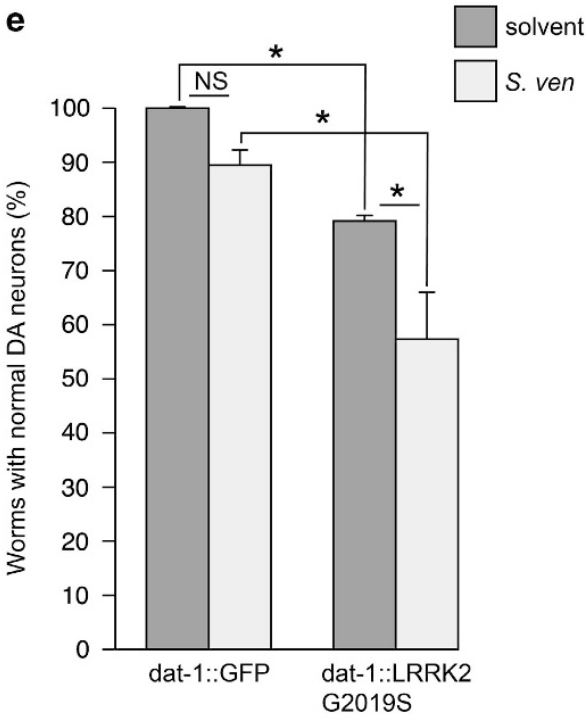

Figure 5 Gene and environment interaction enhances DA neurodegeneration. A combination of exposure to $S$. venezuelae and overexpression of known Parkinson's gene products, $\alpha$-syn (a-d) or LRRK2 G2019S (e) enhances DA neurodegeneration. (a) C. elegans expressing GFP alone exhibit metabolite-induced age-dependent DA neurodegeneration that is evident when examining day 6 versus day 8 animals. Worms overexpressing $\alpha$-syn display age-dependent DA neurodegeneration and are also more susceptible to metabolite-induced DA neurotoxicity when compared with populations of $\alpha$-syn-expressing worms treated with solvent only. These data are represented as the mean \pm S.D. $n=90$ per data point $\left({ }^{*} P<0.05\right.$ by one-way ANOVA). (b and $\left.\mathbf{c}\right)$ Representative images of $C$. elegans (strain UA44) expressing $\alpha$-syn and GFP specifically in the six anterior DA neurons in solvent (b) or in combination with metabolite exposure (c). In the images, large arrowheads show intact dopaminergic neuron cell bodies. Arrows indicate areas where dopaminergic neurons have degenerated. (d) RNAi knockdown of gas-1, nuo-1, and mev-1 showed enhanced $\alpha$-syn-induced DA neurodegeneration compared with $\alpha$-syn alone without metabolite exposure. C. elegans (strain UA196) were analyzed at day 6 where data were analyzed as the mean \pm S.D. After metabolite exposure, nuo-1 RNAi-treated worms showed a significant sensitivity in worms expressing $\alpha$-syn compared with EV (RNAi) metabolite-treated worms and nuo-1 (RNAi) solvent control worms, whereas RNAi of gas-1 and mev-1 failed to cause a significant degeneration with metabolite treatment when compared with untreated controls ( ${ }^{*} P<0.05$; one-way ANOVA). (e) Metabolite-treated worms expressing GFP alone do not display a significant DA neurodegeneration when compared with solvent control at 7 days of exposure. Susceptibility to metabolite-induced DA neurotoxicity is enhanced when C. elegans overexpress human LRRK2 G2019S when compared with populations of worms treated with solvent only. Data are represented as the mean \pm S.D., $n=90$ per independent transgenic line; where three separate transgenic lines were analyzed $\left({ }^{*} P<0.05\right.$ by one-way ANOVA). Magnification bar $=50 \mu \mathrm{m}$

Defects in mitochondria have long been known to contribute to neurodegeneration. Mitochondrial damage is more deleterious in neurons than other cell types as they are non-mitotic, have high metabolic activity, and low antioxidant capacity. ${ }^{37}$ Using an oxidative stress inducible reporter, sod-3::GFP, we determined that the $S$. venezuelae metabolite increased ROS in vivo by measuring GFP levels within intestinal cells. These data are correlated with ROS production within whole animal extracts, as assayed biochemically using DCF-DA, where
ROS was also upregulated in response to metabolite exposure. Finally, since we were interested in knowing whether the neurotoxicity associated with the metabolite was related to ROS, we treated $C$. elegans with an antioxidant, probucol, and assayed for DA neurodegeneration following metabolite exposure. Probucol protected these animals from neurodegeneration, thus providing further evidence that the metabolite elicits its toxicity at least partly through increased ROS production. 
An accumulation of ROS can trigger the UPR ${ }^{\mathrm{mt}}$, a stressresponse pathway that activates transcription of mitochondrial chaperone genes to promote protein homeostasis. The metabolite-induced activation of the UPR ${ }^{\mathrm{mt}}$ that we observed using a worm strain expressing $h s p-6:$ :GFP was suggestive of a disturbance of mitochondrial homeostasis. Importantly, we previously demonstrated that the metabolite failed to activate hsp-4::GFP, an indicator of endoplasmic reticulum-induced UPR (UPR ${ }^{\mathrm{ER}}$ ). ${ }^{12}$ Thus, our findings suggest that the UPR response triggered by $S$. venezuelae metabolite is mitochondrial specific. The mitochondrial protein-folding environment

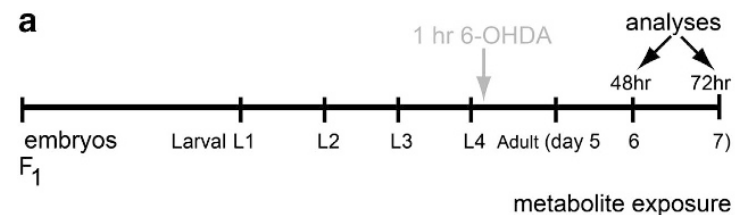

b

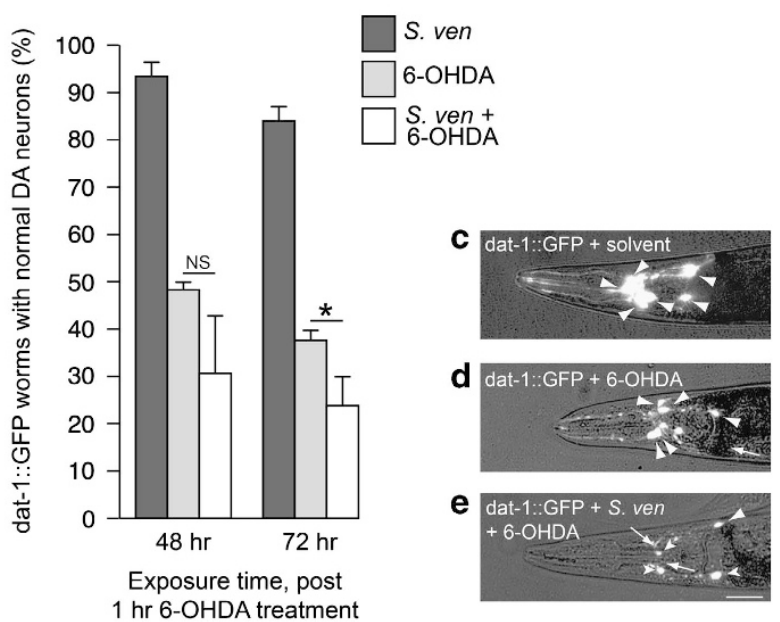

f

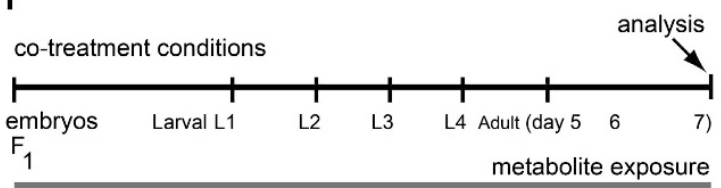

rotenone exposure

g

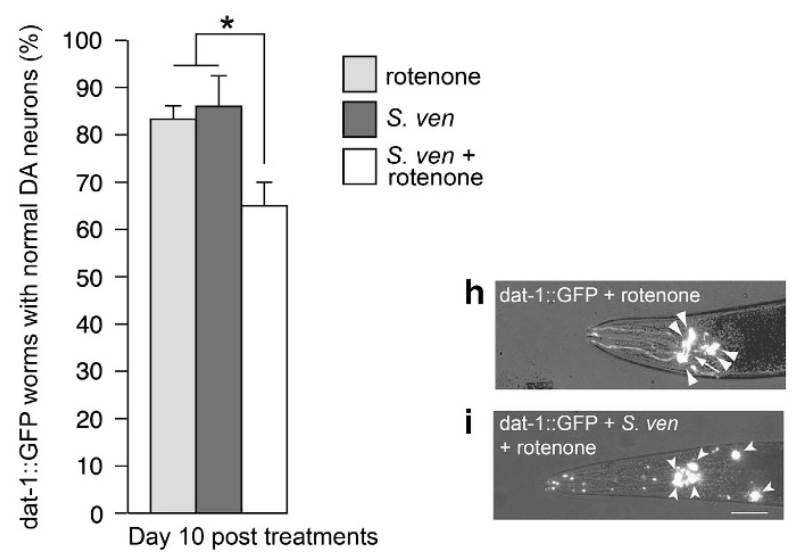

can be perturbed by any changes in the organelle structure, excess production of free radicals, and/or improper function of the electron transport chain. ${ }^{21}$ The neurotoxicity we document in $C$. elegans is coincident with increased ROS generation and depletion of ATP levels, both of which imply mitochondrial dysfunction (Figure 7).

More detailed investigation of the mitochondrial impairment caused by the $S$. venezuelae metabolite highlighted impairment of complex I. Using activators of mitochondrial complex I, riboflavin and $\mathrm{D} \beta \mathrm{HB}, \mathrm{DA}$ neurodegeneration resulting from the metabolite was rescued. Riboflavin has been shown to improve both complex I and complex IV (cytochrome c oxidase) activity ${ }^{28,38}$ whereas $\mathrm{D} \beta \mathrm{HB}$ rescues complex I through complex II. ${ }^{27} \mathrm{D} \beta \mathrm{HB}$ has been also shown to protect mouse DA neurons by mitigating the detrimental effects of complex I inhibition. ${ }^{27}$ Worms treated with RNAi to knockdown complex I genes and the metabolite did not display neurodegeneration, further suggesting that the metabolite might target mitochondrial complex I. However, further investigation will include the direct measurement of complex I-IV enzymatic activities to confirm the direct target, or targets, of the metabolite. Currently, the compound we are utilizing in our studies could be a mixture of more than one metabolite. Efforts are underway to purify the neurotoxic molecule for future investigations.

Current literature suggests a reciprocal relationship between mitochondria and the ubiquitin proteasome system (UPS). ${ }^{39}$ Metabolic function of the UPS impacts the regulation of mitochondrial dynamics, wherein functional perturbations in one of these systems affect the other. More specifically,

Figure 6 Hypersensitivity to $S$. venezuelae DA neurotoxicity when worms are treated with rotenone or 6-OHDA. (a) A timeline representing an experimental paradigm depicting the length of $S$. venezuelae metabolite exposure and 6-OHDA treatment. The abbreviations $\mathrm{L} 1-\mathrm{L} 4$ are the larval stages of $C$. elegans, while the 'adult' designations represent days post hatching. The 48 and $72 \mathrm{~h}$ represents times, post $1 \mathrm{~h} 6-\mathrm{OHDA}(30 \mathrm{mM})$ treatment when DA neurons were analyzed. (b) At $48 \mathrm{~h}$ after 6-OHDA treatment, co-treatment with metabolite was not significantly different from individual treatments alone. Whereas, after $72 \mathrm{~h}$, C. elegans co-exposed with the metabolite and 6-OHDA displayed significantly more susceptibility to DA neurodegeneration than either treatment alone ${ }^{*} P<0.05$; one-way ANOVA; $n=90$ per treatment). (c-e) Representative images of C. elegans (strain BY200) expressing GFP specifically in the six anterior DA neurons. In the images, large arrowheads show intact dopaminergic neuron cell bodies. Arrows indicate areas where dopaminergic neurons have degenerated. Small arrowheads indicate cell body degeneration. (c) A control worm exposed to EtAc solvent only (no 6-OHDA or metabolite) has six normal DA neurons in the anterior region. (d) This representative worm exposed to 6-OHDA is missing one neuron. (e) In this example, a worm exposed to both 6-OHDA and the metabolite is missing two neurons while another three neurons display cell body rounding, indicative of degeneration. Magnification bar $=50 \mu \mathrm{m}$. (f) A timeline representing the experimental paradigm for a combination of $S$. venezuelae metabolite and rotenone exposure scored for DA neurodegeneration. The abbreviations are described in (a). (g) C. elegans co-exposed with the metabolite and $5 \mu \mathrm{M}$ rotenone (in $0.05 \%$ DMSO) display significantly more susceptibility to DA neurodegeneration than either treatment alone ( ${ }^{\star} P<0.01$; Student's $t$-test; $n=90$ per treatment). (h and i) Representative images of $C$. elegans (strain BY200) expressing GFP specifically in the six anterior DA neurons. In the images, large arrowheads show intact dopaminergic neuron cell bodies. Arrows indicate areas where dopaminergic neurons have degenerated. Small arrowheads indicate cell body degeneration. (h) This representative worm exposed to rotenone was missing one neuron. (i) In this representative worm exposed to both rotenone and the metabolite, all neurons display cell body rounding, indicative of degeneration. Magnification bar $=50 \mu \mathrm{m}$ 


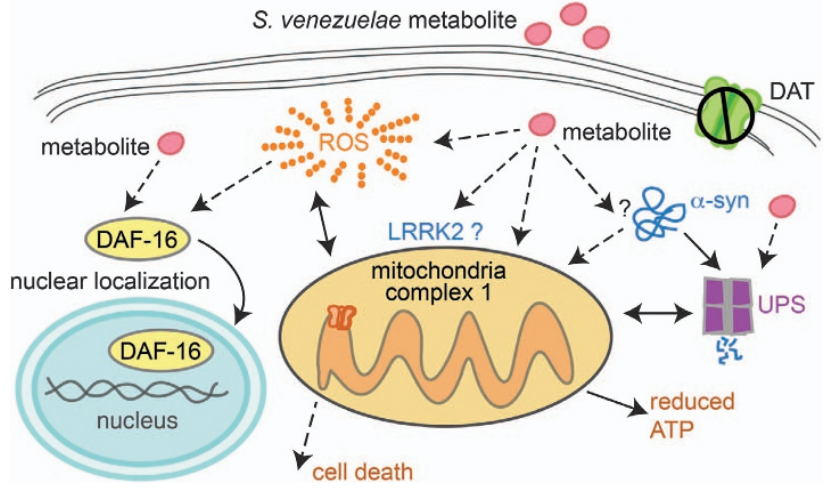

Figure 7 Experimental model for $S$. venezuelae metabolite-induced toxicity. This tentative model depicts our current understanding of the cellular mechanisms impacted. We previously determined that this metabolite does not enter cell membranes through the DA transporter, DAT. ${ }^{12}$ It is possible that the metabolite diffuses through the cellular membrane because the initial biochemical structural characterization and purification efforts have shown that the molecule is small and highly lipophilic. ${ }^{12}$ However, an unknown receptor could also be transducing a signal cascade. Regardless, we observe enhanced oxidative stress in response to metabolite exposure. The excessive production of ROS by the metabolite could be a direct result of redox cycling in vivo or it could be the result of mitochondrial dysfunction. There could also be a reciprocal relationship between ROS and mitochondrial dysfunction following metabolite exposure. Alternatively, the metabolite could directly target mitochondrial complex I, leading to ATP production impairment, upregulation of the UPR ${ }^{\mathrm{mt}}$. These cellular responses could trigger cell death. Additionally, the metabolite could activate the FOXO transcription factor DAF-16 directly or as a secondary cellular response to ROS production. Furthermore, based on our DA neurodegeneration studies, there might be a direct or indirect association between the $S$. venezuelae metabolite and $\alpha$-synuclein or LRRK2 in targeting mitochondria. We have previously reported that the metabolite inhibits the UPS. ${ }^{12}$ Considering that proteasome function intersects with the various mechanisms described in this model, future studies will explore this functional association.

mitochondrial complex I activity has been linked with UPS function. ${ }^{40}$ Previously, we have reported that $S$. venezuelae metabolite inhibits the UPS. ${ }^{12}$ Thus, the bacterial metabolite seems to be involved in several intersecting pathways associated with neurodegeneration, including oxidative stress, mitochondrial dysfunction, and proteasomal dysfunction/protein aggregation. The exact mechanistic order underlying toxicity is yet to be determined. It is possible that UPS inhibition is either a consequence of direct proteasome inhibition by the metabolite or is indirectly caused by mitochondrial impairment or oxidative damage from ROS. Conversely, proteasome inhibition might in turn affect mitochondrial respiration (Figure 7). It is notable that in our genetic studies the effect of the metabolite appears to be enhanced in the presence of $\alpha$-syn. Complex I and II gene knockdowns in an $\alpha$-syn sensitized background uncovered a hypersensitivity of the complex I component, nuo-1, to the metabolite, suggesting that DA neurons are more susceptible to degenerative stressors. Thus, under conditions where protein homeostasis is already out of balance, a direct or indirect relationship between the bacterial metabolite and $\alpha$-syn may result in mitochondrial dysfunction.

Oxidative stress and mitochondrial dysfunction are also two inseparable elements of PD, where mitochondria are a common target of several genetic and environmental risk factors. In this regard, our results on the combined neurotoxic effect of 6-OHDA, rotenone, or familial risk factors for PD with the $S$. venezuelae metabolite support a multiple-hit contribution to dopaminergic neurodegeneration. For example, others and we have shown a relationship between $\mathrm{Mn}++$ toxicity, $\alpha$-syn misfolding, and lysosomal dysfunction associated with the ATP13A2/PARK9 gene product. ${ }^{41}$ Similarly, overexpression of LRRK2 mutations in $C$. elegans is associated with changes in mitochondrial function and autophagy. ${ }^{42}$ In this study, we found metabolite-enhanced neurodegeneration of $C$. elegans expressing LRRK2 G2019S. This was reminiscent of results in other model organisms where expression of mutant LRRK2 is associated with increased sensitivity to rotenone. ${ }^{43}$ Several familial forms of PD have identified gene mutations that cause mitochondrial dysfunction, including parkin, PINK1, and DJ-1; studies designed to evaluate combined impact of the metabolite with these mutations are in progress.

In considering potential mechanisms that could trigger an oxidative stress response, we discerned that the $S$. venezuelae metabolite caused the FOXO transcription factor protein, DAF-16, to translocate to the nucleus in a manner similar to what has been reported in response to paraquat. ${ }^{44}$ We conclude that this nuclear accumulation caused by the metabolite relates to increased intracellular ROS and may be under the control of a genetic program to combat pathogens, sense mitochondrial dysfunction or promote cell death. ${ }^{13,45-47}$ DAF-16, along with SKN-1, are transcription factors that work in parallel and are directly inhibited by the insulin-signaling pathway. Each transcription factor contributes to stress resistance and has a set of target genes, some of which are overlapping. It is interesting to note that following exposure to the $S$. venezuelae metabolite, C. elegans expressing a reporter for SKN-1 did not exhibit activation, in contrast to results reported for exposure to paraquat. ${ }^{48}$ While beyond the scope of this study, future research will explore DAF-16 targets of the metabolite. $A$ recent study described a genome-wide RNAi screen for gene products that were upregulated in a $\mathrm{UPR}^{\mathrm{mt}}$ readout in C. elegans following paraquat exposure. ${ }^{49}$ Considering that most, but not all cellular readouts are identical following metabolite and paraquat treatments in C. elegans, it would be interesting to examine newly identified regulators of ROS-induced UPR ${ }^{\mathrm{mt}}$.

In conclusion, we report that the $S$. venezuelae metabolite shares common molecular mechanisms with other PD toxicants that cause DA neurodegeneration, such as impairment of mitochondrial complex 1 and upregulation of the $\mathrm{UPR}^{\mathrm{mt}}$. These studies advance our understanding of DA neurodegeneration and as well provide an additional model for analysis of PD-associated pathogenesis.

\section{Materials and Methods}

C. elegans strains. Nematodes were maintained using standard procedures. $^{50}$ We obtained the following strains from CGC: TJ356 [zls356

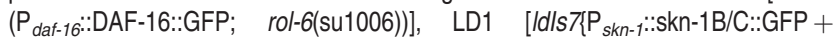

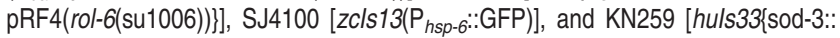
GFP + pRF4(rol-6(su1006))\}]. Strain BY200 [vtls1\{P dat-1::GFP, pRF4

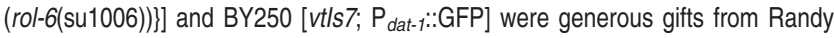

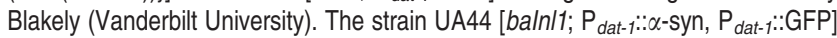
expresses $\alpha$-syn and GFP in the DA neurons. UA202 [sid-1 (pk3321); baln33 ( $\mathrm{P}_{\text {dat }-1: \text { sid-1, }} \mathrm{P}_{\text {myo-2: }: \text { mCherry); }}\left(\mathrm{P}_{\text {dat }-1: \because G F P)]}\right.$ expresses GFP and SID-1 in

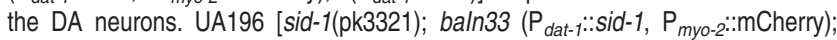


( $P_{\text {dat }-1: \because \alpha-\text { syn, }} P_{\text {dat }-1:: G F P)]}$ expresses $\alpha$-syn, GFP, and SID- 1 in the DA neurons. ${ }^{30,51}$ UA202 and UA196 strains are sensitive to RNAi specifically in the DA neurons. $P_{\text {dat }-1: \text { LRRK2 }}$ G2019S plasmids were co-injected into the gonads of strain BY250 [vtls7; $P_{\text {dat }-1: \text { GFP] }}$ to generate UA215 [baEx128; $P_{\text {dat }-1 \% \text { :LRRK2 }}$ G2019S; vtls7(P $\mathrm{P}_{\text {dat- } 1 .: \mathrm{GFP})]^{52}}$

Isolation and extraction of $S$. venezuelae metabolite. Spores from $S$. venezuelae strain (ARS NRRL ISP-5230) were inoculated in 5 liters of SYZ media at a density of $1 \times 10^{8}$ and were grown at $30^{\circ} \mathrm{C}$ in a shaker. Samples were harvested at $\sim 20$ days when cell density became constant. Cell debris was removed by centrifugation at $10000 \mathrm{~g}$ for $10 \mathrm{~min}$ and supernatants were sequentially passed through eight PES filter membranes with the following pore sizes: $11,6,2.7,1.7,1.2,0.7,0.45$, and $0.22 \mu \mathrm{m}$. After filtration, the conditioned media was extracted with an equal volume of DCM using a separatory funnel. The mixture was gently shaken and the phases were allowed to separate overnight. The DCM layer was collected and the process was repeated two more times. The organic phases were pooled together, dried, and resuspended in $1 \mathrm{ml}$ of EtAc. For all worm assays, $25 \mu \mathrm{l} / \mathrm{ml}$ of partially purified $S$. venezuelae medium or $25 \mu \mathrm{l} / \mathrm{ml}$ EtAc (solvent control) was added to the surface of nematode growth medium (NGM) Petri plates along with E. coli (strain OP50).

C. elegans neurodegeneration assay. Adult $\mathrm{P}_{\text {dat }-1: \text { GFP animals (strain }}$ BY200 or strain BY250), $P_{\text {dat }-1: \alpha} \alpha$-syn (strain UA44), or $P_{\text {dat }-1 \cdots: \text { LRRK2 G2019S }}$ (strain UA215) was placed on plates with $S$. venezuelae metabolite and allowed to lay eggs for $\sim 4 \mathrm{~h}$, before the adults were removed. The worm embryos were grown under constant exposure to partially purified $S$. venezuelae metabolite or EtAc (solvent control) until the day of analysis. Worms were transferred to freshly made plates every other day and a total of 30-40 worms were scored for neurodegeneration after $6,8,10$, or 12 days of metabolite exposure (3-, 5-, 7-, or 9-day-old adults). Worms were considered as normal when all six anterior dopaminergic neurons (two ADE (anterior deirid) and four CEP (cephalic)) were intact and no visible signs of degeneration were observed. If a worm displayed a neuron with any degenerative change (missing neuronal processes, rounding or cell body loss, or blebbing process), then it was scored as exhibiting a degenerative phenotype. The $\mathrm{P}_{\text {dat }-1 .:}:$ GFP animals (strains BY200 and BY250) and $P_{\text {dat }-1: \alpha} \alpha$-syn (strain UA44) animals have chromosomally integrated transgenes where a single isogenic line was used for analyses in all experiments. The LRRK2 G2019S transgene in strain UA215 remains as extrachromosomal arrays. Therefore, three independent lines of transgenic worms were analyzed and an average of total percentage of worms with neurodegeneration was reported for these experiments.

RNAi treatments for neurodegeneration assay. The mev-1 (T07C4.7), nuo-1 (C09H10.3), gas-1 (K09A9.5), and L4440 (EV control) RNAi feeding constructs were obtained from the $C$. elegans Ahringer library. ${ }^{53}$ These RNAi bacterial feeding clones were isolated and grown overnight in LB media containing $100 \mu \mathrm{g} / \mathrm{ml}$ ampicillin. Small NGM plates (4 $\mathrm{ml}$ worm agar media) containing $1 \mathrm{mM}$ IPTG were seeded with $250 \mu$ l RNAi culture and allowed to dry overnight. Next day, equal concentration $(25 \mu / \mathrm{ml})$ of partial purified S. venezuelae metabolite and EtAc (solvent control) were added to the respective plates. Ten dauer worms (DA neuron-specific RNAi worm strains, UA202 and UA196) were transferred to the plates and grown at $20^{\circ} \mathrm{C}$ for $48 \mathrm{~h}$. Adult worms were then transferred to another set of freshly made RNAi + metabolite or $\mathrm{RNAi}+\mathrm{EtAc}$ plates and allowed to lay eggs for $6 \mathrm{~h}$ to synchronize. The DA neurons in the $F_{1}$ progeny of the RNAi-treated worms were analyzed at day 6 or day 12 for neurodegeneration, as described above.

Semi-acute dosage of animals bearing fluorescent reporters. For sod-3::GFP, hsp-6::GFP, daf-16::GFP and skn-1::GFP reporter assay, a mixture of L3 and L4 staged animals from each genotype were placed onto small NGM plates with $0.4 \% \beta$-lactose and ampicillin plates seeded with $250 \mu$ l RNAi bacteria: L4440 EV as a control or mev-1 (T07C4.7) for UPR stress response assay or daf2 (Y55D5A.5) for oxidative stress assays. These RNAi strains were obtained from Ahringer $C$. elegans library. ${ }^{53}$ Each plate was supplemented with an equal concentration $(25 \mu \mathrm{l} / \mathrm{ml})$ of partial purified $S$. venezuelae metabolite or EtAc (solvent control). This initial propagation is to establish a generational exposure to the metabolite in utero. Worms were then allowed to hatch and synchronize at L1 stage for $10-12 \mathrm{~h}$. Next, animals were collected in $10 \mathrm{ml}$ conical glass tubes, washed with M9 buffer and spun down at $10000 \mathrm{~g}$ for $10 \mathrm{~min}$. After the supernatant was removed, a final concentration $(25 \mu / \mathrm{ml})$ of metabolite or EtAc was added to the tubes, followed by gentle shaking for $8 \mathrm{~h}$. This treatment establishes an acute pulse of dosage followed by chronic exposure. The treated worms were placed on respective RNAi plates and were analyzed at $60 \mathrm{~h}$ post treatment (young adult worms).

Quantification of ROS. Intracellular ROS were measured in C. elegans using DCF-DA (C-369, Life Technologies, Carlsbad, CA, USA) as described previously. ${ }^{15}$ Stock solution of $20 \mathrm{mM}$ DCF-DA dissolved in DMSO was stored in $-20^{\circ} \mathrm{C}$. A total of 30 age-synchronized wild-type worms were collected in microcentrifuge tubes after 7,10, and 12 days of EtAc or metabolite or $100 \mu \mathrm{M}$ paraquat exposure. After washing with $\mathrm{M} 9$ buffer (for $1 \mathrm{l}: 3 \mathrm{~g} \mathrm{KH}_{2} \mathrm{PO}_{4}, 6 \mathrm{~g}$ $\mathrm{Na}_{2} \mathrm{HPO}_{4}, 5 \mathrm{~g} \mathrm{NaCl}, 1 \mathrm{ml} \mathrm{MgSO}_{4}$ ) the worms were suspended into $100 \mu \mathrm{l}$ of PBS with 1\% Tween-20 (PBST). The worms were then subjected to repeated freezethaw cycling and sonication to rupture the outer cuticle. The worm lysates were then collected to 96 -well plates. A final concentration of 50 nM DCF-DA in $100 \mu \mathrm{l}$ PBS was added to each well. ROS-associated fluorescence levels were measured kinetically using SpectraMax M2e Microplate Reader (Molecular Devices, Sunnyvale, CA, USA) at excitation wavelength of $485 \mathrm{~nm}$ and emission wavelength of $530 \mathrm{~nm}$, room temperature, every $20 \mathrm{~min}$ for $2.5 \mathrm{~h}$. Data were normalized to solvent control. ROS measurements were conducted on three independent replicates and are presented as means \pm S.D.

6-OHDA and rotenone assay. 6-OHDA assay was performed as previously described. ${ }^{54}$ Metabolite- and EtAc-treated L4 worms (BY200) were washed with $\mathrm{ddH}_{2} \mathrm{O}$ three times, and treated with $30 \mathrm{mM} \mathrm{6-OHDA}$ (Tocris Bioscience, Bristol, UK) containing $1 \mathrm{mM}$ ascorbic acid, followed by gentle agitation for $1 \mathrm{~h}$. Subsequently, the worms were again washed and put onto freshly made metabolite or EtAc plates until analysis. For rotenone assay, BY200 worms were exposed to the metabolite or EtAc until $L 4$ and then transferred to plates containing metabolite, along with $5 \mu \mathrm{M}$ of rotenone or DMSO (as a solvent control for rotenone).

ATP measurements. The ATP assay was performed as described before, ${ }^{55}$ with minor modifications. Worms were grown on metabolite or EtAc and collected for assay. As a positive control, EtAc-treated worms were soaked in $1 \mathrm{mM} \mathrm{MPP}+$ (Sigma, Santa Ana, CA, USA) for $1 \mathrm{~h}$, left on seeded plates for $4-5 \mathrm{~h}$ to recover and then collected. In all, 100 age-synchronized young adult wild-type worms were washed with M9 buffer, treated with three free-thaw cycles and boiled for $15 \mathrm{~min}$ to release ATP and destroy ATPase activity. Samples were then spun at $4^{\circ} \mathrm{C}$, $11000 \mathrm{~g}$ for $10 \mathrm{~min}$. A Life Technologies ATP determination kit (Molecular Probes, Eugene, OR, USA, A22066), which utilizes luciferase to catalyze the formation of light from ATP-dependent oxidation of D-luciferin, was used to quantify ATP contents. ATP concentrations were determined using standard curve derived from bioluminescence of known ATP concentrations. A single-tube luminometer (GloMax 20/20; Promega, Madison, WI, USA) was used to measure levels of bioluminescence. For normalization, protein levels were determined by a BCA protein assay kit (Pierce, Thermo Scientific, Rockford, IL, USA).

Drug treatment. A final concentration of $100 \mu \mathrm{M}$ Paraquat (Sigma), $1 \mu \mathrm{g} / \mathrm{ml}$ Riboflavin (Calbiochem, EMD Bioscience, San Diego, CA, USA), $1 \mathrm{mM}$ Probucol (MP Biomedicals, St. Louis, MO, USA), and $50 \mathrm{mM} \mathrm{D} \beta \mathrm{HB}$ (Sigma-Aldrich, St. Louis, MO, USA) were supplemented in the worm media for their respective experiments. The $S$. venezuelae metabolite or EtAc was added to these plates before use.

Quantitative fluorescence measurements and statistics. Worms were immobilized with $3 \mathrm{mM}$ levamisole and mounted on $2 \%$ agarose pads on a microscope slide. Fluorescent microscopy was performed using a Nikon Eclipse E800 epifluorescence microscope equipped with an Endow GFP HYQ filter cube (Chroma Technology, Bellows Falls, VT, USA). A Cool Snap CCD camera (Photometrics, Tucson, AZ, USA) driven by the MetaMorph software (Molecular Devices) was used to acquire images. Each animal was imaged in the same region at the same magnification and exposure intensity as previously described. ${ }^{56}$ When analyzing each animal, a $100 \times 100 \mu \mathrm{m}$ box was used in the same region of the animal. For the sod-3 transcriptional fusion reporter, this area was the region at the anterior bulb of the pharynx. For the $h s p-6$ transcriptional fusion reporter, this area was the intestinal lumen immediately posterior to the grinder of the pharynx. Pixel intensity was quantified in this manner and compiled across 
three-four separate replicates. For DAF-16 experiment, the entire animal was observed. If no nuclear accumulation could be seen, then it was scored as cytoplasmic, if some, accumulation was observed then it was scored as both, and if robust, nuclear accumulation could be observed throughout the animal then it was scored as nuclear. For the SKN-1 experiment, a dual red-green filter was used to observe emerging green fluorescence from the intestine over the background fluorescence of gut granules (which appear yellow). A similar scoring system was used to that of DAF-16. For statistical analysis, a one-way ANOVA followed by post hoc Tukey's or Dunnett's test was employed for comparison of more than two data sets (Prism 6.0 software; GraphPad, La Jolla, CA, USA). For comparisons between two data sets, Student's t-test was performed. All the experiments were performed with three independent replicates and are presented as means \pm S.D. A value of $P \leq 0.05$ or $P \leq 0.01$ is considered as statistically significant.

\section{Conflict of Interest}

The authors declare no conflict of interest.

Acknowledgements. We would like to thank all members of the Caldwell laboratory for their creative and collegial nature. We would also like to thank Julie Olson, Tyler Hodges, Janna Brown, and Robert Findlay for collaborative assistance in prior experimental acquisition and partial purification of the $S$. venezuelae metabolite. The Caenorhabditis Genetics Center (CGC), which is funded by $\mathrm{NIH}$ Office of Research Infrastructure Programs (P40 OD010440), provided C. elegans strains. This research was funded by NIH grant R15NS074197-01 to KAC.

1. Spillantini MG, Schmidt ML, Lee VM, Trojanowski JQ, Jakes R, Goedert M. Alphasynuclein in Lewy bodies. Nature 1997; 388: 839-840.

2. Tanner $\mathrm{CM}$. Is the cause of Parkinson's disease environmental or hereditary? Evidence from twin studies. Adv Neurol 2003; 91: 133-142.

3. Priyadarshi A, Khuder SA, Schaub EA, Priyadarshi SS. Environmental risk factors and Parkinson's disease: a meta-analysis. Environ Res 2001; 86: 122-127.

4. Costello S, Cockburn M, Bronstein J, Zhang X, Ritz B. Parkinson's disease and residential exposure to maneb and paraquat from applications in the central valley of California. Am J Epidemiol 2009; 169: 919-926.

5. Liou HH, Tsai MC, Chen CJ. Environmental risk factors and Parkinson's disease: a casecontrol study in Taiwan. Neurology 1997; 48: 1583-1588.

6. Greenamyre JT, MacKenzie G, Peng TI, Stephans SE. Mitochondrial dysfunction in Parkinson's disease. Biochem Soc Symp 1999; 66: 85-97.

7. Gorell JM, Johnson CC, Rybicki BA, Peterson EL, Richardson RJ. The risk of Parkinson's disease with exposure to pesticides, farming, well water, and rural living. Neurology 1998 50: 1346-1350.

8. Yano K, Yokoi K, Sato J, Oono J, Kouda T, Ogawa Y et al. Actinopyrones A, B and C, new physiologically active substances. II. Physico-chemical properties and chemical structures. $J$ Antibiot 1986; 39: 38-43.

9. Thiffault C, Langston JW, Di Monte DA. Increased striatal dopamine turnover following acute administration of rotenone to mice. Brain Res 2000; 885: 283-288.

10. Lotharius J, O'Malley KL. The Parkinsonism- inducing drug 1-methyl-4-phenylpyridinium triggers intra- cellular dopamine oxidation: a novel mechanism of toxicity. J Biol Chem 2000; 275: 38581-38588.

11. Cocheme HM, Murphy MP. Complex I is the major site of mitochondrial superoxide production by paraquat. J Biol Chem 2008; 283: 1786-1798.

12. Caldwell KA, Tucci ML, Armagost J, Hodges TW, Chen J, Memon SB et al. Investigating bacterial sources of toxicity as an environmental contributor to dopaminergic neurodegeneration. PLoS One 2009; 4: e7227.

13. Essers MAG, Vries-Smits LMM, Barker N, Polderman PE, Burgering BMT, Korswagen HC. Functional interaction between $\beta$-Catenin and FOXO in oxidative stress signaling. Science 2005; 308: 1181-1184

14. Libina N, Berman JR, Kenyon C. Tissue-specific activities of C. elegans DAF-16 in the regulation of lifespan. Cell 2003; 115: 489-502.

15. Wu Y, Wu Z, Butko P, Christen Y, Lambert MP, Klein WL et al. Amyloid-3-induced pathological behaviors are suppressed by Ginkgo biloba extract EGb 761 and Ginkgolides in transgenic Caenorhabditis elegans. J Neurosci 2006; 26: 13102-13113.

16. Honda $Y$, Honda $S$. The daf-2 gene network for longevity regulates oxidative stress resistance and Mn-superoxide dismutase gene expression in Caenorhabditis elegans. FASEB J 1999; 13: 1385-1393.

17. Henderson ST, Johnson TE. daf-16 integrates developmental and environmental inputs to mediate aging in the nematode Caenorhabditis elegans. Curr Biol 2001; 11: 1975-1980.

18. Motohashi H, Yamamoto M. Nrf-2-Keap-1 defines a physiologically important stress response mechanism. Trends Mol Med 2004; 10: 549-557.
19. An JH, Blackwell TK. SKN-1 links C. elegans mesendodermal specification to a conserved oxidative stress response. Genes Dev 2003; 17: 1882-1893.

20. Hoeven Rv McCallum KC, Cruz MR, Garsin DA. Ce-Duox1/BLI-3 generated reactive oxygen species trigger protective SKN-1 activity via p38 MAPK signaling during infection in C. elegans. PLoS Pathog 2011; 7: e1002453.

21. Haynes CM, Ron D. The mitochondrial UPR- protecting organelle protein homeostasis. $J$ Cell Sci 2010; 123: 3849-3855.

22. Yoneda T, Benedetti C, Urano F, Clark SG, Harding HP, Ron H. Compartment-specific perturbation of protein handling activates genes encoding mitochondrial chaperones. J Cell Sci 2004; 117: 4055-4066.

23. Durieux J, Wolff S, Dillin A. The cell non-autonomous nature of electron transport chain-mediated longevity. Cell 2011; 144: 79-91

24. Wang YM, Pu P, Le WD. ATP depletion is the major cause of MPP + induced dopamine neuronal death and worm lethality in alpha-synuclein transgenic C. elegans. Neurosci Bull 2007; 23: 329-335.

25. Glinka YY, Youdim MB. Inhibition of mitochondrial complexes I and IV by 6-hydroxydopamine. Eur J Pharmacol 1995; 292: 329-332.

26. Betarbet R, Sherer TB, MacKenzie G, Garcia-Osuna M, Panov AV, Greenamyre JT. Chronic systemic pesticide exposure reproduces features of Parkinson's disease. Nat Neurosci 2000; 3: 1301-1306.

27. Tieu K, Perier C, Caspersen C, Teismann P, Wu DC, Yan SD et al. D-beta-hydroxybutyrate rescues mitochondrial respiration and mitigates features of Parkinson disease. J Clin Invest 2003; 112: 892-901.

28. Grad LI, Lemire BD. Mitochondrial complex I mutations in Caenorhabditis elegans produce cytochrome coxidase deficiency, oxidative stress and vitamin-responsive lactic acidosis. Hum Mol Genet 2004; 13: 303-314.

29. Ved R, Saha S, Westlund B, Perier C, Burnam L, Sluder A et al. Similar patterns of mitochondrial vulnerability and rescue induced by genetic modification of $\alpha$-Synuclein, Parkin, and DJ-1 in Caenorhabditis elegans. J Biol Chem 2005; 280: 42655-42668.

30. Harrington AJ, Yacoubian TA, Slone SR, Caldwell KA, Caldwell GA. Functional analysis of VPS41-mediated neuroprotection in Caenorhabditis elegans and mammalian models of Parkinson's disease. J Neurosci 2012; 32: 2142-2153.

31. Devi L, Raghavendran V, Prabhu BM, Avadhani NG, Anandatheerthavarada HK. Mitochondrial import and accumulation of alpha-synuclein impair complex I in human dopaminergic neuronal cultures and Parkinson disease brain. J Biol Chem 2008; 283: 9089-9100.

32. Hamamichi S, Rivas RN, Knight AL, Cao S, Caldwell KA, Caldwell GA. Hypothesis-based RNAi screening identifies neuroprotective genes in a Parkinson's disease model. Proc Natl Acad Sci 2008; 105: 728-733.

33. Nakamura K, Nemani VM, Azarbal F, Skibinski G, Levy JM, Egami K et al. Direct membrane association drives mitochondrial fission by the Parkinson disease-associated protein $\alpha$-Synuclein. J Biol Chem 2011; 286: 20710-20726.

34. Mortiboys $\mathrm{H}$, Johansen $\mathrm{KK}$, Aasly JO, Bandmann O. Mitochondrial impairment in patients with Parkinson disease with the G2019S mutation in LRRK2. Neurology 2010; 75: 2017-2020.

35. Saha S, Guillily MD, Ferree A, Lanceta J, Chan D, Ghosh J et al. LRRK2 modulates vulnerability to mitochondrial dysfunction in C. elegans. J Neurosci 2009; 29: 9210-9218.

36. Cohen G, Heikkila RE. The generation of hydrogen peroxide, superoxide radical, and hydroxyl radical by 6-hydroxydopamine, dialuric acid, and related cytotoxic agents. J Bio Chem 1974; 249: 2447-2452.

37. Exner N, Lutz AK, Haass C, Winklhofer KF. Mitochondrial dysfunction in Parkinson's disease: molecular mechanisms and pathophysiological consequences. EMBO J 2012; 31: 3038-3062.

38. Grad LI, Lemire BD. Riboflavin enhances the assembly of mitochondrial cytochrome c oxidase in C. elegans NADH-ubiquinone oxidoreductase mutants. Biochim Biophys Acta 2006; 1757: 115-122

39. Radke S, Chander H, Schäfer P, Meiss G, Krüger R, Schulz JB et al. Mitochondrial protein quality control by the proteasome involves ubiquitination and the protease Omi. J Biol Chem 2008; 283: 12681-12685.

40. Hoglinger GU, Carrard G, Michel PP, Medjaà F, Lombesà A, Ruberg M et al. Dysfunction of mitochondrial complex I and the proteasome: interactions between two biochemical deficits in a cellular model of Parkinson's disease. J Neurochem 2003; 86: 1297-1307.

41. Gitler AD, Chesi A, Geddie ML, Strathearn KE, Hamamichi S, Hill KJ et al. Alpha-synuclein is part of a diverse and highly conserved interaction network that includes PARK9 and manganese toxicity. Nat Genet 2009; 41: 308-315

42. Di Domenico F, Sultana R, Ferree A, Smith K, Barone E, Perluigi M et al. Redox proteomics analyses of the influence of co-expression of wild-type or mutated LRRK2 and Tau on C. elegans protein expression and oxidative modification: relevance to Parkinson disease. Antioxid Redox Signal 2012; 17: 1490-1506.

43. $\mathrm{Ng} \mathrm{CH}$, Mok SZ, Koh C, Ouyang X, Fivaz ML, Tan EK et al. Parkin protects against LRRK2 G2019S mutant-induced dopaminergic neurodegeneration in Drosophila. J Neurosci 2009; 29: 11257-11262.

44. Kondo M, Yanase S, Ishii T, Hartman PS, Matsumoto K, Ishii N. The p38 signal transduction pathway participates in the oxidative stress-mediated translocation of DAF-16 to Caenorhabditis elegans nuclei. Mech Ageing Dev 2005; 126: 642-647.

45. Chavez V, Mohri-Shiomi A, Maadani A, Vega LA, Garsin DA. Oxidative stress enzymes are required for DAF-16 mediated immunity due to generation of reactive oxygen species in Caenorhabditis elegans. Genetics 2007; 176: 1567-1577. 
46. Lehtinen MK, Yuan Z, Boag PR, Yang Y, Villén J, Becker EB et al. A conserved MST-FOXO signaling pathway mediates oxidative stress responses and extends lifespan. Cell 2006; 125: 987-1001.

47. Jacobs KM, Pennington JD, Bisht KS, Aykin-Burns N, Kim HS, Mishra M et al. SIRT3 interacts with the daf-16 homolog FOXO3a in the mitochondria, as well as increases FOXO3a dependent gene expression. Int J Biol Sci 2008; 4: 291-299.

48. Tullet JMA, Hertweck M, An JH, Baker J, Hwang JY, Liu S et al. Direct inhibition of the longevity promoting factor SKN-1 by Insulin-like signaling in C. elegans. Cell 2008; 132: 1025-1038.

49. Runkel ED, Liu S, Baumeister R, Schulze E. Surveillance-activated defenses block the ROS-induced mitochondrial unfolded protein response. PLOS Genet 2013; 3 : e10003346.

50. Brenner S. The genetics of Caenorhabditis elegans. Genetics 1974; 77: 71-94.

51. Calixto A, Chelur D, Topalidou I, Chen X, Chalfie M. Enhanced neuronal RNAi in C. elegans using SID-1. Nat Methods 2010; 7: 554-559.

52. Liu Z, Hamamichi S, Lee BD, Yang D, Ray A, Caldwell GA et al. Inhibitors of LRRK2 kinase attenuate neurodegeneration and Parkinson-like phenotypes in C. elegans and Drosophila Parkinson's disease models. Hum Mol Genet 2011; 20: 3933-3942.
53. Kamath RS, Fraser AG, Dong Y, Poulin G, Durbin R, Gotta M et al. Systematic functional analysis of the Caenorhabditis elegans genome using RNAi. Nature 2003; 421: 231-237.

54. Nass R, Hall DH, Miller DM, Blakely RD. Neurotoxin-induced degeneration of dopamine neurons in Caenorhabditis elegans. PNAS 2002; 99: 3264-3269.

55. Yang W, Hekimi S. A. Mitochondrial Superoxide Signal Triggers Increased Longevity in Caenorhabditis elegans. PLoS Biol 2010; 8: e1000556.

56. Kimura K, Tanaka N, Nakamura N, Takano S, Ohkuma S. Knockdown of mitochondrial heat shock protein 70 promotes Progeria-like phenotypes in Caenorhabditis elegans. J Biol Chem 2007; 282: 5910-5918.

(c) (i) $(9)($ Cell Death and Disease is an open-access journal published by Nature Publishing Group. This work is licensed under the Creative Commons Attribution-NonCommercial-No Derivative Works 3.0 Unported License. To view a copy of this license, visit http://creativecommons.org/licenses/by-nc-nd/3.0/ 